Background: The ACR-1997, SLICC-2012 and EULAR/ACR-2019 classification criteria have high sensitivity and specificity for SLE, yet they classify non-overlapping groups of patients suggesting that they can be supplemented with additional features to improve their diagnostic performance.

Objectives: To identify criteria and non-criteria manifestations that are significantly associated with SLE in clinical practice and can be used to complement the existing sets of classification criteria.

Methods: Individual items from all three classification criteria (ACR-1997, SLICC-2012, EULAR/ACR-2019) and non-criteria features were analyzed in a randomly selected sample of 800 adults diagnosed with SLE or control rheumatologic diseases (1:1 ratio). The classification performance of each set of criteria was analyzed in combination with complementary features; multivariable least absolute shrinkage and selection operator (LASSO) logistic regression was performed for feature selection. We calculated the diagnostic odds ratio (DOR) of the criteria and the additional features retained in each model.

Results: The EULAR/ACR-2019 and SLICC-2012 criteria have increased accuracy for SLE classification as compared to the ACR-1997 criteria (univariate DOR: 243.2 and 157.3 versus 78.8 , respectively). In multivariable regression based on the ACR-1997 criteria, inclusion of additional features such as maculopapular rash, alopecia and hypocomplementemia significantly enhanced the model predictive capacity (area under the curve [AUC]: 0.95 versus 0.87 of the ACR-1997 criteria alone). Similar analysis based on the SLICC-2012 and EULAR/ACR-2019 criteria identified photosensitivity as an additional criterion significantly associated with SLE (multivariable DOR: 5.4 and 9.4, respectively). Accordingly, models including photosensitivity had superior predictive capacity over the criteria-only models (AUC: 0.94 versus 0.91 for SLICC-2012, 0.96 versus 0.91 for EULAR/ACR-2019). Furthermore, non-criteria features including Raynaud's/livedo reticularis, anti-RNP antibodies, splenomegaly and myocarditis were independently associated with SLE thus enhancing further the predictive capacity of criteria-based models.

Conclusion: We identified a number of criteria and non-criteria features which can be used in combination with the existing sets of criteria to increase classification of SLE patients in clinical practice. Photosensitivity could be considered as an additional feature to improve sensitivity of the recent classification criteria.

Disclosure of Interests: Christina Adamichou: None declared, Irini Genitsaridi: None declared, Dionysis Nikolopoulos: None declared, Alessandra Bortoluzzi: None declared, Antonis Fanouriakis Paid instructor for: Paid instructor for Enorasis, Amgen, Speakers bureau: Paid speaker for Roche, Genesis Pharma, Mylan, Eleni Kalogiannaki: None declared, Emmanouil Papastefanakis: None declared, Irini Gergianaki: None declared, Prodromos Sidiropoulos: None declared, Dimitrios Boumpas: None declared, George Bertsias Grant/research support from: GSK, Consultant of: Novartis

DOI: 10.1136/annrheumdis-2020-eular.6103

\section{THU0246 \\ DIAGNOSTIC CLUSTER PROFILING OF PATIENTS IN A REAL-WORLD DATA SET WITH SYSTEMIC LUPUS ERYTHEMATOSUS}

Z. Touma ${ }^{1}$, B. Hoskin ${ }^{2}$, C. Atkinson ${ }^{2}$, D. Bell ${ }^{2}$, O. Massey ${ }^{2}$, J. H. Lofland ${ }^{3}$, P. Berry ${ }^{3}$, C. Karyekar ${ }^{3}$, K. Costenbader ${ }^{4} .{ }^{1}$ University of Toronto, Toronto, Canada; ${ }^{2}$ Adelphi Real World, Cheshire, United Kingdom; ${ }^{3}$ Global Commercial Strategic Organisation, Johnson and Johnson, Horsham, United States of America; ${ }^{4}$ Brigham and Women's Hospital, Boston, United States of America

Background: Previous systemic lupus erythematosus (SLE) studies have identified potential clusters of SLE clinical manifestations post diagnosis.

Objectives: To describe the presentation of SLE at diagnosis across different cohorts of patients and describe management and outcomes after diagnosis within clusters.

Methods: Cross-sectional study of 263 rheumatologists in the US and EU5. Data were collected from the Adelphi Real World 2015 Lupus Disease Specific Programme. Rheumatologists completed patient record forms (PRFs) for the next 5 prospectively consulting SLE patients; these patients completed patient self-completion (PSC) forms describing how SLE affected them. PRF data includes patient's characteristics and management history. PSCs focused on similar data collection, including patient reported outcome measures on the humanistic burden. Age at diagnosis, symptoms at diagnosis, organ involvement at diagnosis, and severity at diagnosis were used as covariates in a latent cluster analysis.

Results: Data were extracted from 1376 PRFs. Cluster analysis resulted in up to 6 clusters, and disease understanding led to the selection of a 4-cluster solution. Cluster 1 displayed the mildest disease, characterised by joint involvement, while cluster 2 displayed more skin involvement in conjunction with joint. Cluster 3 were characterised by renal involvement and cluster 4 had skin and joint involvement, but also high constitutional and haematological involvement at diagnosis (Table 1).
Table 1 Cluster analysis

\begin{tabular}{|c|c|c|c|c|c|c|}
\hline \multirow[b]{2}{*}{$\begin{array}{l}\text { Organ involvement at } \\
\text { diagnosis, } \mathrm{n}(\%)\end{array}$} & \multicolumn{6}{|c|}{ Clusters } \\
\hline & $\begin{array}{l}\text { Overall } \\
(n=1304)\end{array}$ & $\begin{array}{c}1 \\
(n=210)\end{array}$ & $\begin{array}{c}2 \\
(\mathrm{n}=493)\end{array}$ & $\begin{array}{c}3 \\
(n=162)\end{array}$ & $\begin{array}{c}4 \\
(n=439)\end{array}$ & p-value \\
\hline Musculoskeletal & 1145 (87.8) & 174 (82.9) & ) 444 (90.1) & $134(82.7)$ & 393 (89.5) & ) 0.0065 \\
\hline Mucocutaneous & 898 (68.9) & $5(2.4)$ & $397(80.5)$ & 95 (58.6) & 401 (91.3) & $<0.0001$ \\
\hline Neuropsychiatric & $87(6.7)$ & $19(9.0)$ & $9(1.8)$ & $16(9.9)$ & $43(9.8)$ & $<0.0001$ \\
\hline Cardiorespiratory & $176(13.5)$ & $36(17.1)$ & $14(2.8)$ & 22 (13.6) & 104 (23.7) & ) $<0.0001$ \\
\hline Gastrointestinal & $44(3.4)$ & $8(3.0)$ & $14(2.8)$ & $8(4.9)$ & $14(3.2)$ & 0.6115 \\
\hline Opthalmic & 47 (3.6) & 0 & $20(4.1)$ & $10(6.2)$ & $17(3.9)$ & 0.0102 \\
\hline Renal & $213(16.6)$ & $15(7.1)$ & $9(1.8)$ & $162(100)$ & $27(6.2)$ & $<0.0001$ \\
\hline Constitutional & 425 (32.6) & 45 (21.4) & 89 (18.1) & $55(34.0)$ & $236(53.8)$ & ) $<0.0001$ \\
\hline Haematological & $452(34.7)$ & $64(30.5)$ & $22(4.5)$ & $80(49.4($ & $(286(65.1)$ & $<0.0001$ \\
\hline \multicolumn{7}{|c|}{ Severity at diagnosis, $n(\%)$} \\
\hline Mild & 209 (16.0) & $55(26.2)$ & $99(20.1)$ & $1(0.6)$ & 54 (12.3) & $<0.0001$ \\
\hline Moderate & $806(61.8)$ & $122(58.1)$ & $324(65.7)$ & 75 (46.3) & $285(64.9)$ & \\
\hline Severe & 289 (22.2) & $33(15.7)$ & $70(14.2)$ & $86(53.1)$ & $100(22.8)$ & \\
\hline
\end{tabular}

Significant between-cluster differences were observed when comparing outcomes; cluster 4 have been diagnosed longest (mean weeks diagnosed 354.6 v. 1: $232.6,2: 228.7,3: 338.2, p<0.0001)$. Cluster 3 consulted more in the last 12 months (mean number of visits 7.9 vs. $1: 5.7,2: 6.3,4: 7.6$ ).

Significant differences were also observed between clusters in relation to current treatment proportions: corticosteroid (highest cluster 3: 78.4\%), immunosuppres sant (highest cluster 3: 75.3\%), biologic DMARD (highest cluster 4: 17.8\%) and antidepressant (highest cluster 4: 4.1\%).

Conclusion: This study demonstrates the heterogeneity of SLE at diagnosis and highlights four distinct presentations of the disease at diagnosis. Significant proportions of patients present with advanced disease, these clusters go on to present the greatest burden demonstrating the need for better diagnostic tools and novel earlier intervention.

Study funded by Johnson and Johnson.

Disclosure of Interests: Zahi Touma Consultant of: Consultant for Janssen, Ben Hoskin Consultant of: Consultant for Janssen, Christian Atkinson Consultant of: Consultant for Janssen, David Bell Consultant of: Janssen, Olivia Massey Consultant of: Janssen, Jennifer H. Lofland Employee of: Janssen, Pamela Berry Employee of: Janssen, Chetan Karyekar Shareholder of: Johnson \& Johnson, Consultant of: Janssen, Employee of: Janssen Globa Services, LLC. Previously, Novartis, Bristol-Myers Squibb, and Abbott Labs. Karen Costenbader Grant/research support from: Merck, Consultant of: Astra-Zeneca

DOI: 10.1136/annrheumdis-2020-eular.5865

\section{THU0247 \\ FREQUENCY AND PREDICTORS OF THE LUPUS LOW DISEASE ACTIVITY STATE IN CHINESE PATIENTS WITH SYSTEMIC LUPUS ERYTHEMATOSUS: AN OBSERVATIONAL COHORT STUDY}

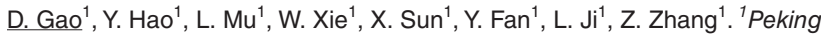
University First Hospital, Rheumatology and Clinical Immunology Department, Beijing, China

Background: As a consensus-based definition of minimally acceptable disease activity in systemic lupus erythematosus (SLE), Lupus Low Disease Activity State (LLDAS) has been well-validated and widely accepted. However, no data about the time to LLDAS in Asian ethnicity has been reported so far.

Objectives: To estimate the time to LLDAS and the predictors of time to LLDAS in our prospective observational cohort of Chinese patients with SLE.

Methods: Patients were from Peking University First Hospital SLE cohort and those having not fulfilled LLDAS at enrolment were included in this study. The time to LLDAS and annual cumulative probabilities of LLDAS achievement were estimated by the Kaplan-Meier approach. The predictors of time to LLDAS were identified by univariate and multivariable Cox proportional hazards.

Results: A total of 574 patients with SLE were included and 435 (75.8\%) of them achieved LLDAS during a median 4.2 years of follow-up. The median time to LLDAS was 19.0 months and the cumulative probabilities at 1, 2, 3, 5 and 10 years were $19.8 \%, 57.6 \%, 72.0 \%, 85.1 \%$ and $98.0 \%$, respectively. In multivariable Cox models, older age at disease onset, treatment-naïve and hydroxychloroquine prescription were found to be independent predictors of shorter time to LLDAS, after adjusted by daily prednisone dose, SLE Disease Activity Index 2000 and physician's global assessment. Finally, we developed a matrix model based on the identified independent predictors to present the time to LLDAS in patients with respective characteristics.

Conclusion: Our study proved that LLDAS is attainable as an early treatment target for SLE in Chinese patients. The older age at disease onset, treatment-naïve and hydroxychloroquine prescription were independent predictors of shorter time to LLDAS. 
References:

[1] Franklyn K, Lau CS, Navarra SV, Louthrenoo W, Lateef A, Hamijoyo L, Wahono CS, Chen SL, Jin O, Morton S, Hoi A, Huq M, Nikpour M, Morand EF; Asia-Pacific Lupus Collaboration. Ann Rheum Dis. 2016 Sep;75(9):1615-21.

Table 1 Baseline variables associated with LLDAS achievement based on multivariable Cox models

\begin{tabular}{|c|c|c|c|c|c|c|}
\hline \multirow[t]{2}{*}{ Characteristics } & \multicolumn{2}{|c|}{ Model 1} & \multicolumn{2}{|c|}{ Model 2} & \multicolumn{2}{|l|}{ Model 3} \\
\hline & HR (95\% Cl) & $p$ value & HR $(95 \% \mathrm{Cl})$ & $p$ value & HR (95\% Cl) & $\begin{array}{c}p \\
\text { value }\end{array}$ \\
\hline $\begin{array}{l}\text { Age at disease onset, } \\
\text { years }\end{array}$ & $\begin{array}{c}1.010 \\
(1.003-1.016)\end{array}$ & 0.005 & $\begin{array}{c}1.009 \\
(1.002-1.016)\end{array}$ & 0.010 & $\begin{array}{c}1.010 \\
(1.003-1.017)\end{array}$ & 0.004 \\
\hline Treatment-naïve & $\begin{array}{c}1.346 \\
(1.105-1.641)\end{array}$ & 0.003 & $\begin{array}{c}1.425 \\
(1.161-1.749)\end{array}$ & 0.001 & $\begin{array}{c}1.484 \\
(1.204-1.830)\end{array}$ & $<0.001$ \\
\hline Nephritis & $\begin{array}{c}0.776 \\
(0.641-0.939)\end{array}$ & 0.009 & $\begin{array}{c}1.066 \\
(0.820-1.385)\end{array}$ & 0.634 & $\begin{array}{c}0.925 \\
(0.737-1.160)\end{array}$ & 0.498 \\
\hline SLEDAI & & & $\begin{array}{c}0.968 \\
(0.950-0.987)\end{array}$ & 0.001 & & \\
\hline PGA & & & & & $\begin{array}{c}0.685 \\
(0.551-0.853)\end{array}$ & 0.001 \\
\hline $\begin{array}{l}\text { Daily prednisone (or } \\
\text { equivalent) dose, } \\
\text { mg/d }\end{array}$ & & & $\begin{array}{c}1.003 \\
(0.998-1.007)\end{array}$ & 0.266 & $\begin{array}{c}1.005 \\
(0.999-1.010)\end{array}$ & 0.093 \\
\hline $\mathrm{HCQ}$ & $\begin{array}{c}1.638 \\
(1.263-2.123)\end{array}$ & $<0.001$ & $\begin{array}{c}1.713 \\
(1.318-2.225)\end{array}$ & $<0.001$ & $\begin{array}{c}1.664 \\
(1.284-2.157)\end{array}$ & $<0.001$ \\
\hline
\end{tabular}

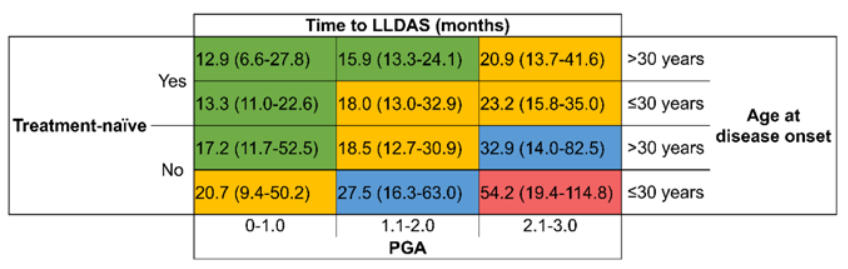

Figure 1 Matrix model for time to LLDAS.

Disclosure of Interests: None declared

DOI: 10.1136/annrheumdis-2020-eular.3149

\section{THU0248 GLOMERULAR AND TUBULOINTERSTITIAL LESIONS IN PER-PROTOCOL REPEAT BUT NOT BASELINE KIDNEY BIOPSY PORTEND RELAPSE AND LONG-TERM RENAL FUNCTION IMPAIRMENT, RESPECTIVELY, IN INCIDENT CASES OF PROLIFERATIVE LUPUS NEPHRITIS}

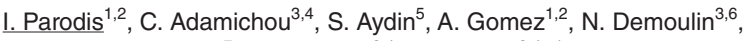
J. Weinmann-Menke ${ }^{7}$, F. Houssiau ${ }^{3,4}$, F. Tamirou ${ }^{3,4}$. ${ }^{1}$ Karolinska Institutet, Department of Medicine Solna, Stockholm, Sweden; ${ }^{2}$ Karolinska University Hospital, Rheumatology, Stockholm, Sweden; ${ }^{3}$ Université Catholique de Louvain, Institut de Recherche Expérimentale et Clinique, Brussels, Belgium; ${ }^{4}$ Cliniques Universitaires Saint-Luc, Rheumatology Department, Brussels, Belgium; ${ }^{5}$ Cliniques Universitaires Saint-Luc, Pathology Department, Brussels, Belgium; ${ }^{6}$ Cliniques Universitaires Saint-Luc, Division of Nephrology, Brussels, Belgium; ${ }^{7}$ University Medical Centre of the Johannes Gutenberg University of Mainz, Department of Nephrology, Rheumatology and Clinical Immunology, Mainz, Germany

Background: In patients with lupus nephritis (LN), clinical response to treatment and renal histopathology have been shown to be discordant. No clinical or laboratory markers have to date been shown to reliably portend renal prognosis, in particular renal function impairment.

Objectives: To investigate whether per-protocol repeat renal biopsies are predictive of $L N$ relapses and long-term impairment of renal function.

Methods: Forty-two patients with an incident biopsy-proven active proliferative (class III/IV $\pm \mathrm{V}$ ) $L N$ from the $L N$ database of the Universite catholique de Louvain were included in the present retrospective study. Per-protocol repeat kidney biopsies were performed in all patients after a median time of 24.3 (IQR: 21.3-26.2) months. The $\mathrm{NIH}$ activity index (AI) and chronicity index (Cl) scores were assessed in both baseline and repeat biopsies. We defined acute glomerular lesions as cellular proliferation, fibrinoid necrosis or karyorrhexis, cellular crescents, hyaline thrombi or wire loops, and leucocyte infiltration, and chronic glomerular lesions as glomerular sclerosis and fibrous crescents, in alignment with the NIH activity and chronicity indices. Similarly, we defined acute tubulointerstitial lesions as mononuclear cell infiltration and chronic tubulointerstitial lesions as interstitial fibrosis and tubular atrophy.

Results: Despite a moderate correlation between urinary protein/creatinine $(\mathrm{U}-\mathrm{P} / \mathrm{C})$ ratios and $\mathrm{Al}$ scores at repeat biopsy $(\mathrm{r}=0.48$; $\mathrm{P}=0.001)$, ten patients $(23.8 \%)$ with $\mathrm{U}-\mathrm{P} / \mathrm{C}$ ratios $<1.0 \mathrm{~g} / \mathrm{g}$ still had a high degree of histological activity (Al score $>3$ ). High Al scores in repeat (but not baseline) kidney biopsies were associated with an increased probability and/or shorter time to renal relapse $(\mathrm{N}=11)$ following the repeat biopsy (HR: $1.2 ; 95 \% \mathrm{Cl}: 1.1-1.3 ; \mathrm{P}=0.007)$, independently of proteinuria levels. This association remained significant for the $\mathrm{NIH}$ activity index items within the glomerular but not the tubulointerstitial compartment of the kidney biopsies. High $\mathrm{NIH} \mathrm{Cl}$ scores in repeat (but not baseline) kidney biopsies were associated with a sustained increase in serum creatinine levels corresponding to $\geq 120 \%$ of the baseline value (HR: $1.8 ; 95 \% \mathrm{Cl}: 1.1-2.9$; $\mathrm{P}=0.016$ ) through a median follow-up time of 131.5 (IQR: 73.8-178.2) months, being the case also for acute and chronic tubulointerstitial lesions in repeat but not baseline kidney biopsies.

Conclusion: Our results highlight the usefulness of per-protocol repeat biopsies as an integral part of the treatment evaluation, also in patients who have shown adequate clinical response. Glomerular lesions consistent with active renal disease portend $\mathrm{LN}$ relapses, while tubulointerstitial lesions consistent with active disease and chronic damage portent long-term renal function impairment.

Disclosure of Interests: Ioannis Parodis: None declared, Christina Adamichou: None declared, Selda Aydin: None declared, Alvaro Gomez: None declared, Nathalie Demoulin: None declared, Julia Weinmann-Menke: None declared, Frederic Houssiau Grant/research support from: UCB, Consultant of: GSK, Farah Tamirou: None declared DOI: 10.1136/annrheumdis-2020-eular.5863

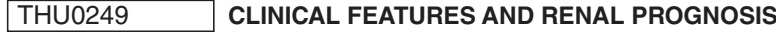 IN LUPUS NEPHRITIS PATIENTS UNDERGOING A REPEATED BIOPSY: RESULTS OF 103 RE-BIOPSIES IN 438 PATIENTS}

M. Gatto ${ }^{1}$, F. Saccon ${ }^{1}$, F. Radice ${ }^{2}$, P. G. Vercelloni ${ }^{2}$, R. A. Sinico ${ }^{2}$, G. Frontini ${ }^{3}$, V. Binda ${ }^{3}$, P. Messa ${ }^{3}$, F. Alberici ${ }^{4}$, G. Moroni ${ }^{3}$, A. Doria ${ }^{1}$. ${ }^{1}$ University of Padova, Unit of Rheumatology - Department of Medicine, Padova, Italy; ${ }^{2}$ Università Milano Bicocca - ASST Monza - S Gerardo, Nefrologia, Monza, Italy; ${ }^{3}$ Fondazione IRCCS Ca Granda Ospedale Maggiore Policlinico, Nefrologia, Milano, Italy; ${ }^{4}$ ASST Santi Paolo e Carlo, Ospedale San Carlo Borromeo, Nefrologia, Milano, Italy

Background: Indications to repeat renal biopsy (RB) in lupus nephritis (LN) are not unanimously acknowledged.

Objectives: To evaluate the renal outcome of patients with $\mathrm{LN}$ undergoing a second RB.

Methods: We retrospectively analyzed prospectively collected data of patients with LN followed up in four Italian referral centres for systemic lupus eryhtematosus. Serological and clinical information were retrieved according to a shared database. RB were classified according to ISN/RPS 2003 classification; chronicity $(\mathrm{Cl})$ and activity indexes (AI) were defined according to Austin et al. The primary renal outcome was renal failure, defined as serum creatinine $(\mathrm{SCr})>1.0 \mathrm{mg} / \mathrm{dL}$ with $\mathrm{eGFR}<60 \mathrm{ml} / \mathrm{min}$. Non-parametric tests were used for statistics. Patients repeating RB due to renal remission were excluded from the analysis.

Results: Four-hundred and thirty-eight patients were recruited. One-hundred and three patients repeated RB after $6.1 \pm 4.7$ (mean $\pm S D$ ) years from the first due to: protocol biopsy due to renal remission (Group 1, $n=8$ ); proteinuric flare (Group 2, $n=51$ ); worsened renal function (Group 3, $n=26$ ); partial renal response (Group $4 \mathrm{n}=18)$. Patients undergoing a second RB were younger $(\mathrm{p}<0.001)$, had lower serum $C 3$ at $L N$ diagnosis $(p<0.001)$ and displayed more frequently class IV and higher Al at first RB ( $p=0.0038$ and $p=0.043$, respectively). At the end of follow-up, patients who repeated RB had more frequently renal failure $(p=0.003)$. At the second RB, the histological class was unchanged in $55 \%$ of patients. $\mathrm{Cl}$ increased at second RB compared to the first $(3.6 \pm 2.4$ vs. $1.7 \pm 1.7 ; p<0.001)$ Overall, 26 out of 103 patients (25\%) developed renal failure: 0 from group 1, 10 from group 2, 14 from group 3, 2 from group $4(p<0.001)$. Uncontrolled hypertension at LN diagnosis, increased $\mathrm{SCr}$ and increased proteinuria at second RB predicted renal failure (Table)

Conclusion: Patients undergoing a repeated RB had more aggressive clinical and histological features already at first RB and developed renal failure more frequently. Among baseline features, uncontrolled hypertension had the strongest association with renal failure, thus suggesting that control of blood pressure since early stages is highly advisable.

References:

[1] Austin HA, et al. Predicting renal outcomes in severe lupus nephritis: contributions of clinical and histologic data. Kidney Int 1994;45:544-50. 